\title{
Statusbericht - H2020-ROBOMINERS und M30 Projekttreffen
}

\author{
Jasmin Leitner, Michael Berner und Nikolaus August Sifferlinger
}

Lehrstuhl für Bergbaukunde, Bergtechnik und Bergwirtschaft, Montanuniversität Leoben, Leoben, Österreich

Eingegangen 14. Dezember 2021; angenommen 10. Januar 2022; online publiziert 26. Januar 2022

Zusammenfassung: Das H2020 Robominer Projekt ist ein EU gefördertes Projekt, und das Konsortium dafür besteht aus 14 verschiedenen Projektpartnern. Im Zuge dieses Projektes hat in Leoben ein persönliches Treffen mit den Projektpartnern stattgefunden, um die bisherigen Entwicklungen des Projektes zu präsentieren, zu besprechen und einen Bergbau in Slowenien für potenzielle Feldversuche zu besuchen. Des Weiteren wird ein Überblick über den aktuellen Stand des Projektes gegeben.

Schlüsselwörter: ROBOMINERS, Bergbau, Montanmaschinenbau, Robotik

Status Report-H2020-ROBOMINERS and M30 Project Meeting

Abstract: The H2020 Robominer project is funded by the $\mathrm{EU}$, and the consortium consists of 14 different project partners. In the course of the project, a meeting with all project partners took place in Leoben. The developments of the project were presented, reviewed, and an underground mine for potential field tests was visited. Furthermore, an overview of the current status was given.

Keywords: ROBOMINERS, Mining, Heavy-duty engineering, Robotics

Zum jetzigen Stand ist knapp die Hälfte des ROBOMINERS Projektes absolviert, und das Design vom Prototypen und vom Abbauwerkzeug wurde finalisiert. Der Prototyp hat eine wasserhydraulische Gesamtleistung von $40 \mathrm{~kW}$ und kann sich durch vier Schraubenräder, welche der archimedischen Schraube gleichen, fortbewegen. Über wasserhydraulische Zylinder und künstliche Muskeln können diverse Manipulationen vorgenommen werden.

J. Leitner, B.Sc. $(\varangle)$

Lehrstuhl für Bergbaukunde, Bergtechnik und Bergwirtschaft,

Montanuniversität Leoben,

Franz-Josef-Straße 18,

8700 Leoben, Österreich

jasmin.leitner@unileoben.ac.at
Die Funktionsweise des Roboters besteht darin, die Umgebung zu scannen und zur Ortsbrust zu navigieren. Dort angekommen, scannt er die erste Gesteinsschicht, welche sich direkt dahinter befindet, bis zu einer maximalen Tiefe von $10 \mathrm{~cm}$. Dabei schneidet er mit dem Schneidkopf kontinuierlich Gestein, wobei nur sehr weiches Material, aufgrund des geringen Gewichts und der geringen Leistung, schneidbar ist. Danach wird das abgebaute Material aufgeschlämmt und abgepumpt. Im Inneren des Roboters befinden sich Sensoren zur Materialanalyse, die die Suspension analysieren. In Abb. 1 ist das Sensorsystem abgebildet. Die

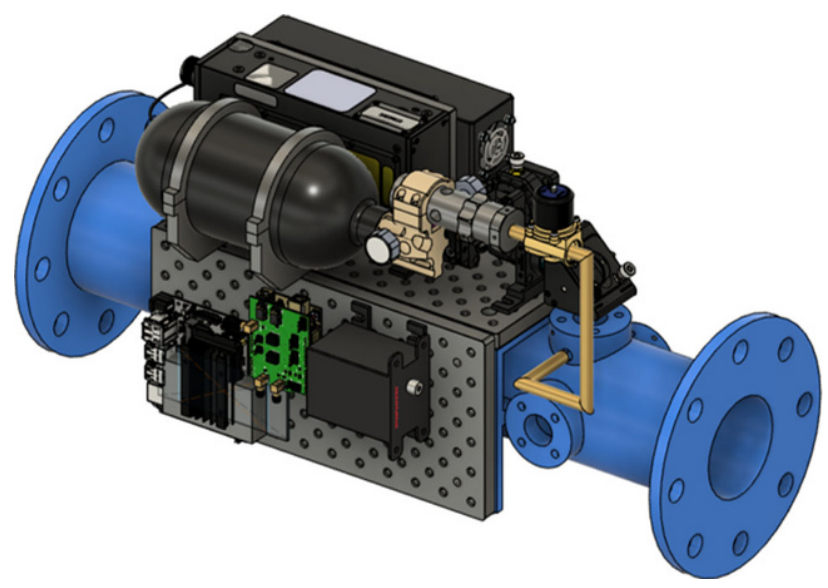

Abb. 1: Sensorsystem zur Materialanalyse

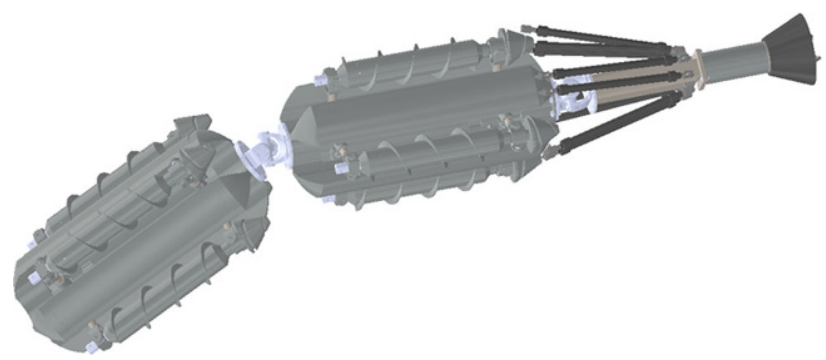

Abb. 2: Aufbau des Prototyps 


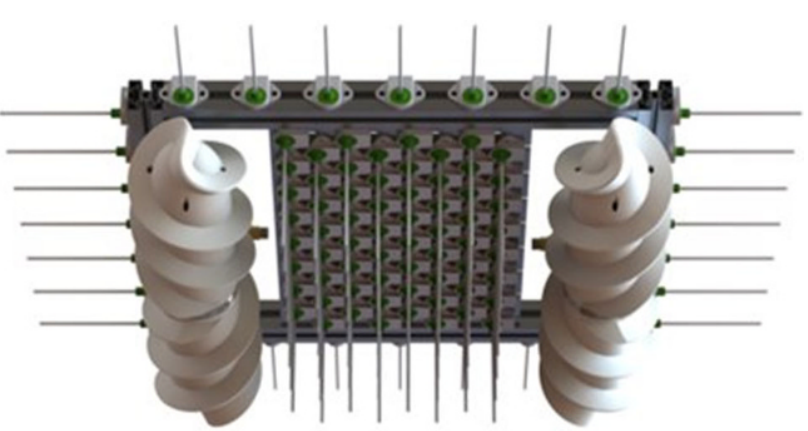

Abb. 3: Simulation der Schraubenräder

Analyse erfolgt mittels der LIBS (Laser Induced Breakdown Spectroscopy) und der UV-Fluoreszenz Methode.

Für Tests wird eine modulare Bauweise verwendet, die aus zwei Modulen und dem Abbauwerkzeug besteht. In Abb. 2 ist der Aufbau des Prototyps des Robominers dargestellt. Zur richtigen Navigation wird der Prototyp mit der notwendigen Sensorik und Tastsensoren ausgestattet. Diese Navigationsausstattung erstellt ein virtuelles 3D Modell der Umgebung und der Oberfläche. Zusätzlich sind Sensoren für die Materialidentifikation und die Analysen verbaut.

Zum aktuellen Zeitpunkt werden die Schraubenräder hinsichtlich ihrer Bodenhaftung und Antriebsleistung getestet. Des Weiteren werden Simulationen und Labortest von der Sensorik durchgeführt, zur Testung und Überprüfung ihrer Funktionsweise. Die eingebauten Tasthaare dienen zur Navigation und zum Erkennen von der Oberflächenbeschaffenheit. Des Weiteren werden dafür auch Radartechnologien und Kameras verwendet. Zur Veranschaulichung der Kinematik und der Interaktion des Roboters mit der Umgebung helfen die erstellten Simulationen. Diese stellen auch die Interaktion mehrerer Module dar. In Abb. 3 ist ein Ausschnitt der simulierten Schraubenräder dargestellt.

Das Abbauwerkzeug ist ein kleiner Axialschneidkopf, dieser ist für sehr weiche Materialien geeignet und weist

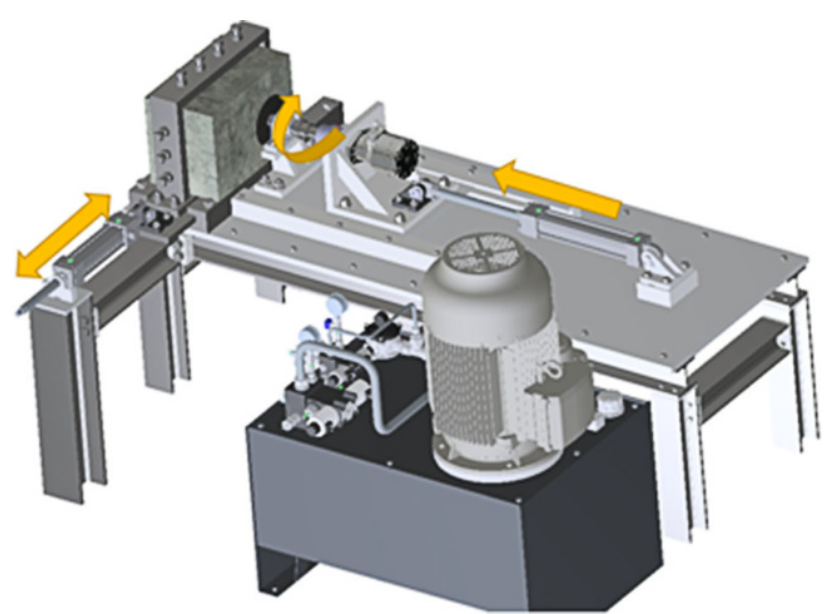

Abb. 4: Prüfstand für den Schneidkopf

eine Antriebsleistung von $17,5 \mathrm{~kW}$ auf. Der Schneidkopf wird gerade auf einem Prüfstand, welcher in Abb. 4 dargestellt ist, getestet, um Aussagen über die auftretenden Schneidkräfte, Vibrationen, den Verschleiß der Meißel und andere geforderte Parameter zu treffen.

Michael Berner begrüßte mit seinem Team die Projektpartner des H2020 Robominer Projektes nach fast zwei Jahren zu einem persönlichen Treffen von 16.11. bis 18.11.2021 in Leoben. Abb. 5 zeigt die Projektpartner beim M30 ROBOMINERS Meeting in Leoben. Durch die hybride Abhaltung des Meetings konnten alle Konsortiums Mitglieder anwesend sein, und es konnten bei dem zweiten Clustering Event weitere externe Personen teilnehmen.

Am ersten Tag wurde am Vormittag der aktuelle Stand der einzelnen Arbeitspakete präsentiert, besprochen und die weiteren Schritte für die kommenden Monate festgelegt. Dabei stellte Michael Berner den Statusbericht zu seinem Arbeitsbereich, die Entwicklung des Abbauwerkzeuges des Robominers, vor.
Abb. 5: Projektpartner bei dem M30 Robominer Meeting

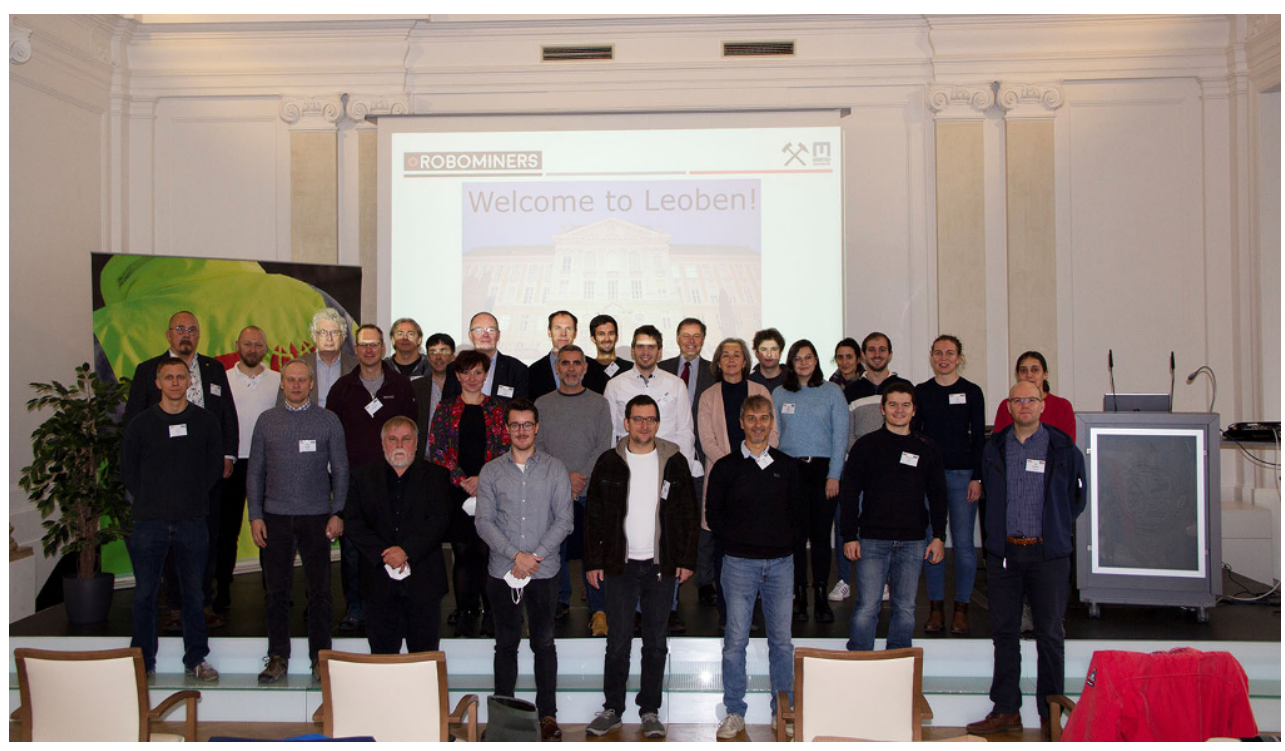


Das Programm für den Nachmittag waren Gruppendiskussionen, bei denen die Präsentationen des Vormittags die Grundlage dafür bildeten. Die Teilnehmer/innen teilten sich abhängig von ihrem Spezialgebiet in Gruppen auf und diskutierten die folgenden Themen:

- Robotik und Sensorik über Abbaumethoden /-technologien

- Gebirgsmechanik

- Software und Simulation

Am zweiten Tag fand ein Ausflug nach Mežica in Slowenien zur Besichtigung eines potenziellen Bergbaus für Feldversuche mit dem Robominer statt. Dieser Bergbau ist nicht mehr aktiv und wird nur noch für Touristenbesuche verwendet. Vor Ort wurden die Teilnehmer/innen in eine Expertenund eine Touristengruppe aufgeteilt. Der Leiter des Bergbaus führte die Expertengruppe durch den Bergbau und zeigte ihnen ein paar Stellen, die für Feldversuche geeignet wären. Die Projektpartner haben einige Gesteinsproben mitgenommen, um das Gestein physikalisch und mineralogisch zu analysieren. Die Touristengruppe bekam ebenfalls eine Führung durch den Bergbau und konnte dort sehen, wie sich die Abbauverfahren von Beginn bis zum Schließen des Bergbaus entwickelt haben. Zum Schluss wurde das angrenzende Museum besichtigt, in dem eine Wohnung von früher nachgestellt wurde und eine Reliefkarte der Umgebung, gezeichnete Pläne sowie sehr viele Mineralien des Bergbaus ausgestellt sind.

Am letzten Tag wurde zu Beginn kurz über den besuchten Bergbau diskutiert und die Vor- und Nachteile bzw. Eignung des Bergbaus für Feldversuche wurden besprochen.
Danach fand das zweite Clustering Event mit dem Titel „The key technological obstacles towards a fully autonomous robotic mining (eco) system" statt. Dieses Clustering Event wurde im Rahmen der EIT Raw Materials Week veranstaltet und konnte sowohl physisch als auch online besucht werden. Dabei wurden neue, innovative und zukunftsorientierte Technologien der Rohstoffwertschöpfungskette vorgestellt und anschließend diskutiert.

Finanzierung. Dieses Projekt wurde vom Forschungs- und Innovationsprogramm Horizon 2020 der Europäischen Union im Rahmen der Fördervereinbarung Nr. 820971 finanziert.

Funding. Open access funding provided by Montanuniversität Leoben.

Open Access Dieser Artikel wird unter der Creative Commons Namens nennung 4.0 International Lizenz veröffentlicht, welche die Nutzung, Vervielfältigung, Bearbeitung, Verbreitung und Wiedergabe in jeglichem Medium und Format erlaubt, sofern Sie den/die ursprünglichen Autor(en) und die Quelle ordnungsgemäß nennen, einen Link zur Creative Commons Lizenz beifügen und angeben, ob Änderungen vorgenommen wurden.

Die in diesem Artikel enthaltenen Bilder und sonstiges Drittmaterial unterliegen ebenfalls der genannten Creative Commons Lizenz, sofern sich aus der Abbildungslegende nichts anderes ergibt. Sofern das betreffende Material nicht unter der genannten Creative Commons Lizenz steht und die betreffende Handlung nicht nach gesetzlichen Vorschriften erlaubt ist, ist für die oben aufgeführten Weiterverwendungen des Materials die Einwilligung des jeweiligen Rechteinhabers einzuholen.

Weitere Details zur Lizenz entnehmen Sie bitte der Lizenzinformation auf http://creativecommons.org/licenses/by/4.0/deed.de.

Hinweis des Verlags. Der Verlag bleibt in Hinblick auf geografische Zuordnungen und Gebietsbezeichnungen in veröffentlichten Karten und Institutsadressen neutral. 